\title{
Some Approximation in Cone Metric Space and Variational Iterative Method
}

\author{
Ning Chen, Jiqian Chen \\ School of Science, Southwest University of Science and Technology, Mianyang, China \\ Email: chenning783@163.com, chenning@swust.edu.cn
}

Received September 23, 2012; revised October 23, 2012; accepted November 1, 2012

\begin{abstract}
In this paper, we give some new results of common fixed point theorems and coincidence point case for some iterative method. By using of variation iteration method and an effective modification of He's variation iteration method discusses some integral and differential equations, we give out some new conclusion and more new examples.
\end{abstract}

Keywords: Cone Metric Space; Common Fixed Point; Effective Variation Iteration Method; Integral-Differential Equation

\section{Introduction}

Fixed point theory has fascinated many researchers since 1922 with the celebrated Banach's fixed point theorem. There exist a fast literature on the topic and this is a very active field of research at present (See [1-7]).

By using same definition and meaning in stating is also looking in [2] and [3] etc. we introducing the following results for needing. For convenience, the authors give the following definition and lemma (see the proof of theorem 3 [2])

Let $E$ always be a real Banach space, and $P$ be the subset of $E, P$ is called a cone, if and only if:

(i) $P$ is closed, nonempty, and $P \neq\{0\}$;

(ii) $a, b \in R, a, b \geq 0, x, y \in P \Rightarrow a x+b y \in P$;

(iii) $x \in P$, and $-x \in P \Rightarrow x=0$.

Given a cone $P \subset E$, we define a partial ordering $\leq$ with respect to $P$ by $x \leq y$ if and only if $y-x \in P$.

We shall write $x<y$ to indicate $x \leq y$ but $x \neq y$ while $x \ll y$ implies $y-x \in$ int $P$, int $P$ denotes the interior of $P$.

Definition 1.1 Let $X$ be a non-empty set in $E$, and suppose the mapping $d: X \times X \rightarrow E$ satisfies:

(i) $0 \leq d(x, y)$ for any $x, y \in X$, and $d(x, y)=0 \Leftrightarrow x=y$;

(ii) $d(x, y)=d(y, x)$ for all $x, y \in X$;

(iii) $d(x, y) \leq d(x, z)+d(z, y)$ for all $x, y, z \in X$.

Then $d$ is cone distance on $X,(X, d)$ is called a cone metric space.

It is obvious that cone metric space generalize the metric spaces.

Definition 1.2 Let $(X, d)$ is said to be a complete cone metric space, if every Cauchy sequence is con- vergent in $X$.

Let $(X, d)$ be a metric space. We denote by $C B(X)$ the family of non-empty closed bounded subset of $X$. Let $H(\cdot, \cdot)$ be the Hausdorff metric on $C B(X)$. That is for

$$
H(A, B)=\max \left\{\sup _{a \in A} d(a, B), \sup _{b \in B} d(A, b)\right\} .
$$

where $d(a, B)=\inf \{d(a, b), b \in B\}$ is the distance from point $a$ to the sub-set $B$. An element $x \in X$ is said to be a fixed point of a multi-valued mapping $T: X \rightarrow 2^{X}$ if $x \in T(X)$.

Lemma 1.3 Suppose that $(X, d)$ be a cone metric space and the mapping $T: X \rightarrow X$ hold the sequence $\left\{x_{n}\right\}$ in $(X, d)$ satisfying the following conditions:

$$
d\left(x_{n+1}, x_{n}\right) \leq L d\left(x_{n}, x_{n-1}\right),(0<L<1),
$$

and that is

$$
d\left(x_{n+1}, x_{n}\right) \leq L^{n} d\left(x_{1}, x_{0}\right) .
$$

Then the sequence $\left\{x_{n}\right\}$ is a Cauchy sequence in $(X, d)$.

\section{Several Common Fixed Point Theorems}

In recent years, the fixed point theory and application has rapidly development. Huang and Zhang [2] introduced the concept of a cone metric space that a element in Banach space equipped with a cone which induces a nature order partial order. In the same work, they investigated the convergence and that inequality and they extend the contractive principle to in partial order set $\mathrm{s}$ with some applications to matrix equations and common solution of 
integral equations.

Such theorems are very important tools for proving the existence and eventually the uniqueness of the solutions to various mathematical models (integral and partial differential equations, variations inequalities etc.).

First, we state following some extend conclusion ([3, 4]). Next, authors consider the variation iterative method to some integral and differential equations, and effective method ([5-8]) for examples and numerical test as some Fig case.

Now we first give common fixed point Theorem in similar method for two operators to extend Theorem 2.1 [2] with one operator case. Assume that $(X, d)$ be a complete cone metric space.

Theorem 2.1 Let $(X, d)$ be a complete cone metric space, $P$ a normal cone with normal constant $K$. Suppose that mappings $S, T: X \rightarrow X$ satisfies the Contractive condition

$$
\begin{aligned}
d(S x, T y) \leq & k(d(S x, x)+d(T y, y)) \\
& +\alpha \frac{d(x, T y)}{1+d^{2}(x, T y)} d(x, y)
\end{aligned}
$$

for each $x, y \in X$, where $k \in[0,1 / 2)$ is a constant $(0<\alpha<2)$, Then $S, T$ has a unique common fixed point in $X$. And so for any $x \in X$, the iterative sequence $\left\{T^{n} x\right\}$ con- verges to the fixed point.

Corollary 2.2 Let $\alpha=0$, and $T=S$ then we obtain that theorem 2.1 in [2].

In the same way, authors can extend theorem 4 [2], and omit again these stating.

\section{Some Notes of Common Fixed Point}

The common fixed point theorems for two operators in cone metric space are given in [3]. By using of needing same definition and as same results in it, we consider fixed point theorem in cone metric space to complete and extending the Theorem 1 in it.

Theorem A (See Theorem 1 [3]) Assume that $(X, d)$ be a complete cone metric space. Let mappings

$S, T: X \rightarrow X$ satisfying following Lipchitz conditions for any $x, y \in X$,

$$
\begin{aligned}
& d(S x, T y) \leq A(x, y) d(x, y) \\
& +B(x, y) d(x, S x)+C(x, y) d(y, T y) \\
& +D(x, y) d(x, T y)+E(x, y) d(y, S x),
\end{aligned}
$$

where $A, B, C, D, E, F$ are nonnegative real value functions on $X \times X$, such that

$$
\begin{aligned}
& A(x, y)+D(x, y)+E(x, y)<1, C(x, y)+D(x, y) \\
& <1, B(x, y)+E(x, y)<1
\end{aligned}
$$

and that

$$
\begin{aligned}
& L_{1}=\sup _{x, y \in X} \frac{A(x, y)+B(x, y)+D(x, y)}{1-C(x, y)-D(x, y)}, \\
& L_{2}=\sup _{x, y \in X} \frac{A(x, y)+C(x, y)+E(x, y)}{1-B(x, y)-E(x, y)}, \\
& L_{3}=\sup _{x, y \in X} \frac{1+C(x, y)+D(x, y)}{1-B(x, y)-E(x, y)}, \\
& L_{4}=\sup _{x, y \in X} \frac{1+B(x, y)+E(x, y)}{1-C(x, y)-D(x, y)}, K=L_{2} L_{2}<1 .
\end{aligned}
$$

Then there is a unique common fixed point in $u \in X$ for $S$ and $T$, and for any $x_{0} \in X$, the iterative sequence $\left\{x_{n}\right\}$ convergent to the common fixed point of $S, T$

$$
\left(x_{2 k+1}=S x_{2 k}, x_{2 k+2}=T x_{2 k+1}, k=0,1,2, \cdots\right) \text {. }
$$

Remark in [3], the example 2 illustrate this effect of meanings with this Theorem (non-expansion mapping, not contractive case that have uniqueness common fixed point). Look for multiple-value mappings in some case [5].

We can easy note Theorem A. Now we give complete this fixed point problem below.

Theorem 3.1 Same as the assume of theorem 1 [3]. Let $(X, d)$ be a complete cone metric space, and there exists positive integer $p, q$ and mappings $S, T: X \rightarrow X$ satisfying following Lipchitz conditions for any $x, y \in X$, that is satisfy following inequality such that

$$
\begin{aligned}
& d\left(S^{p} x, T^{q} y\right) \leq A(x, y) d(x, y) \\
& +B(x, y) d\left(x, S^{p} x\right)+C(x, y) d\left(y, T^{q} y\right) \\
& +D(x, y) d\left(x, T^{q} y\right)+E(x, y) d\left(y, S^{p} x\right),
\end{aligned}
$$

where $A, B, C, D, E$ are non-negative real functions on $X \times X$. If it holds, $S T=T S$.

Then there is a unique common fixed point in $u \in X$ for $S$ and $T$.

Proof By the proof of theorem 1 [3] that we known that $S^{p}$ and $T^{q}$ has a unique common fixed point $u$, and $S^{p}(u)=T^{q}(u)=u$, from

$$
S^{p}(T u)=T\left(S^{p} u\right)=T u, T^{q}(u)=T\left(T^{q} u\right)=T u,
$$

then $T u$ is also common fixed point of $S^{p}$ and $T^{q}$ Hence, we have $T u=u$ by the uniqueness of them. In the same way, we know that $S u=u$. that is, $S u=u$. This $u$ is a common fixed point of $S$ and $T$, and

$$
S^{p}(S u)=S\left(S^{p} u\right)=S u, T^{q}(T u) u=T\left(T^{q} u\right)=T u
$$

We have $S u=T u=u$.

On the other hand, if $S v=T v=v(v \neq u)$, then clearly, $S^{p} v=T^{q} v=v$, in the same way, we know $v=u$ which a contradiction. So, we complete the proof of this 
theorem.

Theorem 3.2 Let $(X, d)$ be a complete cone metric space, and mappings $S, T_{i}: X \rightarrow X(i=1,2, \cdots, m)$ satisfying following Lipchitz conditions for any $x, y \in X$, that is satisfy following inequality ( $A, B, C, D, E$ nonnegative real constant)

$$
\begin{aligned}
& d\left(S x, T_{i} y\right) \leq A d(x, y)+B d(x, S x)+C d\left(y, T_{i} y\right) \\
& +D d\left(x, T_{i} y\right)+E d(y, S x) \text { for any } x, y \in X,
\end{aligned}
$$

and

$$
A+B+C+D+E=1, C>B, E>D \text {, or }(C<B, E<D) \text {. }
$$

If $T_{1}, T_{2}, \cdots, T_{m}$ have not common fixed point each other, then the $S$ exist at least $m$ number fixed points in $X$.

Proof By the theorem 1 [3], we known that $S$ and $T_{i}$ has a unique common fixed point $u_{i}$, that is $S\left(u_{1}\right)=T_{1}\left(u_{1}\right)=u_{1}$, and that in the same way,

$$
S\left(u_{2}\right)=T_{2}\left(u_{2}\right)=u_{2}, \cdots, S\left(u_{m}\right)=T_{m}\left(u_{m}\right)=u_{m} .
$$

Then $S$ have at least $m$ number fixed points.

Corollary 3.3 Let $q=p+1$, more positive integer cases in theorem 3.1.

Remark 3.4 (see corollary 5 [3]) Assume that $(X, d)$ be complete cone metric space, and mappings $S, T: X \rightarrow X$ satisfy following condition ( $A, B, C, D, E$ non-negative real constant):

$$
\begin{aligned}
& d(S x, T y) \leq A d(x, y)+B d(x, S x)+C d(y, T y) \\
& +D d(x, T y)+E d(y, S x) \text { for any } x, y \in X,
\end{aligned}
$$

And

$$
A+B+C+D+E=1, C>B, E>D(\text { or }, C<B, E, D),
$$

then $S, T$ must have uniqueness common fixed point, and for any $x_{0} \in X$, iterative sequence $\left\{x_{n}\right\}$ convergent to the common fixed point

$$
\left(x_{2 k+1}=S x_{2 k}, x_{2 k+2}=T x_{2 k+1}, k=0,1,2, \cdots\right) .
$$

Here example 2 in [5] for non-expansion mappings, also have common fixed point case with important meanings.

\section{Common Fixed Point of Four Mappings}

Many authors have extended the contraction mapping principle in difference direction. Some extension of Banach's fixed point theorem through the rational expression form with it's inequality. The purpose of this section, is to establish some common fixed point theorem for four mappings in this space.

Theorem 4.1 Assume that $(X, d)$ be a complete cone metric space, and mappings $S, T, E, F: X \rightarrow X$ continuous and satisfying following conditions for any

$$
\begin{aligned}
& x, y \in X: E S=S E, F T=T F, \\
& E(X) \subset T(X), F(X) \subset S(X),
\end{aligned}
$$

and that inequality

$$
\begin{aligned}
& d(E x, T y) \\
& \leq \frac{\alpha d(S x, E x)+\gamma d(T y, E x)}{d(S x, F y)+d(T y, E x)+d(S x, T y)} d(T x, F y) \\
& +\beta d(S x, T y), \\
& \text { for any } x, y \in X, S x \neq T y \text { and } \alpha, \beta, \\
& \gamma \geq 0,0<\alpha+\beta+\gamma<1
\end{aligned}
$$

Then $E, F, S$ and $T$ have a unique common fixed point.

Proof Let $x_{0}$ be an arbitrary point of $X$ and from $E(X) \subset T(X)$, we can choose a point $X_{1} \in X$ such that $T x_{1}=E x_{0}$. Also $F(X) \subset S(X)$, we can choose a point $x_{2} \in X$ such that $S x_{2}=S x_{1}$. In general, we can Choose $x_{2 n+1} \in X$ and $x_{2 n+2} \in X$ define a sequence $\left\{x_{n}\right\}$ in $X$. as follows,

$$
\begin{aligned}
& T x_{2 n+1}=E x_{2 n}=y_{2 n}, \\
& S x_{2 n+2}=F x_{2 n+1}=y_{2 n+1}, n=0,1,2, \cdots .
\end{aligned}
$$

Now, by (4.1) we have that

$$
\begin{aligned}
& d\left(T x_{2 n+1}, S x_{2 n+2}\right) \\
& =d\left(E x_{2 n}, F x_{2 n+1}\right)=d\left(y_{2 n}, y_{2 n+1}\right) \\
& \leq \frac{\alpha d\left(S x_{2 n}, E x_{2 n}\right)}{d\left(S x_{2 n}, F x_{2 n+1}\right)+\left(S x_{2 n}, T x_{2 n+1}\right)} d\left(y_{2 n}, y_{2 n+1}\right) \\
& +\beta d\left(y_{2 n-1}, y_{2 n}\right) \\
& \leq(\alpha+\beta) d\left(y_{2 n-1}, y_{2 n}\right) \\
& \leq(\alpha+\beta+\gamma) d\left(y_{2 n-1}, y_{2 n}\right) .
\end{aligned}
$$

Similarly, we have

$$
\begin{aligned}
& d\left(y_{2 n+1}, y_{2 n+2}\right)=d\left(F x_{2 n+1}, E x_{2 n+2}\right) \\
& =d\left(E x_{2 n+2}, F x_{2 n+1}\right) \leq(\alpha+\beta+\gamma) d\left(y_{2 n}, y_{2 n+1}\right) .
\end{aligned}
$$

which implies that

$$
d\left(y_{n+1}, y_{n+2}\right) \leq(\alpha+\beta+\gamma) d\left(y_{n+1}, y_{n}\right), n=0,1,2, \cdots .
$$

Hence, it is well know that $\left\{y_{n}\right\}$ is Cauchy sequence. From the $X$. is complete, then there exists $u \in X$ such that the $\left\{y_{n}\right\}$ convergences to $u$ in $X$. Since $\left\{E x_{2 n}\right\}$ and $\left\{F X_{2 n+1}\right\}$ are subsequences of $\left\{y_{n}\right\}$ then it will convergence to same point $u$.

Next, from these $E, F, S$ and $T$ are continuous maps, we can obtain following results

$$
E\left(S x_{2 n}\right) \rightarrow E u \text { and } F\left(T x_{2 n+1}\right) \rightarrow F u(n \rightarrow \infty) \text {. }
$$

It follows from $E S=S E$ and $F T=T F$, then we have $S\left(E x_{2 n}\right)=E\left(S x_{2 n}\right) \rightarrow E u$, and we get $S u=E u$. In the 
same reason,

$$
T\left(F x_{2 n+1}\right)=F\left(T x_{2 n+1}\right) \rightarrow F u(n \rightarrow \infty),
$$

we again obtain $T u=F u$. By (4.1), if $S u \neq T u$, then

$$
d(E u, F u) \leq \beta d(S u, T u)=\beta d(E u, F u),
$$

this is a contradiction. Hence, we have that $S u=E u=F u=T u$

Let $S u=E u=F u=T u=u^{*}$, then we obtain that

$$
E u^{*}=E(E u)=E(S u)=S(E u)=S u^{*},
$$

and

$$
F u^{*}=F(T u)=T(F u)=T(E u)=T u^{*} .
$$

By (4.1), if $F u \neq u^{*}$, then

$$
\begin{aligned}
& d\left(u^{*}, F u^{*}\right)=d\left(E u, F u^{*}\right) \\
& \leq \beta d\left(S u, T u^{*}\right)=\beta d\left(u^{*}, F u^{*}\right),
\end{aligned}
$$

we get $\beta \geq 1$.

It is a contradiction. Thus, $F u^{*}=T u^{*}=u^{*}$.

Similarly,

$$
\begin{aligned}
& d\left(S u^{*}, u^{*}\right)=d\left(E u^{*}, F u^{*}\right) \\
& \leq \beta d\left(S u^{*}, T u^{*}\right)=\beta d\left(S u^{*}, u^{*}\right),
\end{aligned}
$$

we get $\beta \geq 1$.

It is also a contradiction. Therefore, $S u^{*}=E u^{*}=u^{*}$.
By (4.1), if $E u^{*} \neq F u^{*}$, then

$$
\begin{aligned}
d\left(E u^{*}, F u^{*}\right) & \leq \beta d\left(S u^{*}, T u^{*}\right) \\
& =\beta d\left(E u^{*}, F u^{*}\right),
\end{aligned}
$$

also a contradiction. Thus, we have

$$
S u^{*}=E u^{*}=F u^{*}=T u^{*}=u^{*},
$$

then this implies $u^{*}=E u$ for common fixed point of $E, F, S$ and $T$.

(Uniqueness) Let two points $u^{* *}, u^{*}\left(u^{* *} \neq u^{*}\right)$ be the difference common fixed point of $E, F, S$ and $T$.

By (4.1), we have

$$
\begin{aligned}
& d\left(u^{*}, u^{* *}\right)=d\left(E u^{*}, F u^{* *}\right) \\
& \leq \beta d\left(S u^{*}, T u^{* *}\right)=\beta d\left(u^{*}, u^{* *}\right) .
\end{aligned}
$$

A contradiction in the above same reason, then this implies the uniqueness of common fixed point of $E, F, S$ and $T$.

\section{Remarks}

(i) As $\gamma=0, S=T$, we obtain special case.

(ii) $S=T=I$ (Identity map), $E=F, \gamma=0$, we get some special case.

Theorem 4.2 Assume that $(X, d)$ be a complete cone metric space. Same as theorem 4.1, and satisfies these conditions below

$$
\begin{aligned}
& E S=S E, F T=T F \text {, and } E(X) \subset T(X) \text { and } F(X) \subset S(X) \\
& d\left(E^{p} x, T^{q} y\right) \leq \frac{\alpha d\left(S x, E^{p} x\right)+\gamma d\left(T y, E^{p} x\right)}{1+d\left(S x, F^{q} y\right)+d\left(T y, E^{p} x\right)+d(S x, T y)} d\left(T x, F^{q} y\right)+\beta d(S x, T y),
\end{aligned}
$$

for any

$$
x, y \in X, S x \neq \text { Ty and } \alpha, \beta, \gamma \geq 0,0<\alpha+\beta+\gamma<1
$$

Then $E, F, S$ and $T$ have a unique common fixed point.

Proof By theorem 4.1, we known that there is a unique fixed point $u$ in $X$, and $S u=E^{p} u=F^{q} u=T u=u$.

Obviously, $S u=E u=F u=T u=u$. This completes the proof of theorem 4.2.

\section{Some Notes for Multi-Valued Mappings}

According the direction of [5], we give out some coincidence point theorem of maps to extend theorem 2.1 and theorem 2.3 [5].
Let $\varphi:[0, \infty) \rightarrow[0, \infty)$ be a strictly increasing function such that

(i) $\varphi(0)=0$,

(ii) $0<\varphi(t)<t$ for each $t>0$,

(iii) $\sum_{i=1}^{\infty} \varphi^{n}(t)<\infty$ for each $t \in(0, \infty)$.

Now, we can easy obtain following theorems.

Theorem 5.1 Let $(X, d)$ be a complete metric space and $S, T_{i}: X \rightarrow C B(X)$ be multi-valued maps $(i=1,2, \cdots, m)$ satisfying for each $x, y \in X$,

$$
H\left(S x, T_{i} y\right) \leq \varphi \cdot \max \Phi\left(x, y, S x, T_{i} y\right),
$$

where

$$
\Phi(\cdot)=\left\{d(x, y), d(x, S x), d\left(y, T_{i} y\right),\left(d\left(x, T_{i} y\right)+d(y, S x)\right) / 2\right\},
$$

If $T_{1}, T_{2}, \cdots, T_{m}$ have not common fixed point each other, then there exist at least $m$ number fixed points in
$X$.

Proof From theorem 2.1 [5], we known there exist $p_{1}$ 
in $X$. such that $p_{1} \in S p_{1} \cap T p_{1}$, again for $p_{2}$ in $X$. The same way,

$$
p_{2} \in S p_{2} \cap T p_{2}, \cdots, p_{m} \in S p_{m} \cap T p_{m} .
$$

Since $p_{1}, p_{2}, \cdots, p_{m}$ not equality each other, then $S$ have at least $m$ number fixed point. This completes the proof.

Theorem 5.2 Let $(X, d)$ be a complete metric space and $S, T_{i}: X \rightarrow C B(X)$ be multi-valued maps

$(i=1,2, \cdots, m)$ and $f: X \rightarrow X$ be a map satisfying

(i) $T_{i}(X) \subset f(X)$ and $S(X) \subset f(X)$,

(ii) $f(X)$ is complete,

(iii) there exists a function $\phi:(0, \infty) \rightarrow[0,1)$ such that

$$
\lim _{r \rightarrow t^{+}} \sup \phi(r)<1 \text {, for every } t \in[0, \infty) \text {. }
$$

And for each $x, y \in X$,

$$
H\left(S x, T_{i} y\right) \leq \varphi(d(f x, f y)) d(f x, f y) .
$$

If $T_{1}, T_{2}, \cdots, T_{m}$ and $S$ have not coincidence point each other, then the $S$ and $f$ at least exist $m$ number coincidence points in $X$.

Proof From theorem 2.3 [5], we known there exist coincidence point $p_{1}$ in $X$. such that $f p_{1} \in S p_{1} \cap T p_{1}$, in the same way, the coincidence point $p_{2}$ in $X$. with $f p_{2} \in S p_{2} \cap T p_{2}, \cdots, f p_{m} \in S p_{m} \cap T p_{m}$.

Since

$$
p_{1}, p_{2}, \cdots, p_{m}
$$

not equality each other, therefore $S$ and $f$ have at least $m$ number coincidence point. That is,

$$
f p_{i} \in S p_{i} \cap T p_{i},(i=1,2, \cdots, m),
$$

Then this completes the proof.

Corollary 5.3 Let $(X, d)$ be a complete metric space and $T_{i}: X \rightarrow C B(X)$ be multi-valued maps $(i=1,2, \cdots, m)$ and $f: X \rightarrow X$ be a map satisfying

(i) $T_{i}(X) \subset f(X)$;

(ii) $f(X)$ is complete,

(iii) $H\left(T_{i} x, T_{i} y\right) \leq \phi(d(f x, f y)) d(f x, f y)$, for each $x, y \in X(i=1,2, \cdots, m)$, where $\phi:(0, \infty) \rightarrow[0,1)$ such that $\lim _{r \rightarrow t^{+}} \sup \phi(r)<1$, for every $t \in[0, \infty)$.

If $T_{1}, T_{2}, \cdots, T_{m}$ have not coincidence point each other, then $f p_{i} \in S p_{i} \cap T p_{i}(i=1,2, \cdots, m)$.

If we take $f=\mathrm{i} d$, we easy get these conclusion below, where id is the identity map on $X$.

Corollary 5.4 Let $(X, d)$ be a complete metric space and $S, T_{i}: X \rightarrow C B(X)$ be multi-valued maps $(i=1,2, \cdots, m)$ and satisfying for each

$$
x, y \in X, H(S x, T y) \leq \phi(d(x, y)) d(x, y),
$$

where $\phi:(0, \infty) \rightarrow[0,1)$ such that $\lim _{r \rightarrow t^{+}} \sup \phi(r)<1$ for every $t \in[0, \infty)$.

If $T_{1}, T_{2}, \cdots, T_{m}$ have not common fixed point each other, then the $S$ and $T_{i}$ exist at least $m$ number points in $X$. That is case

$$
p_{i} \in S p_{i} \cap T_{i} p_{i},(i=1,2, \cdots, m) \text {. }
$$

Corollary 5.5 When $H\left(T_{i} x, T_{i} y\right) \leq \phi \cdot(d(x, y)) d(x, y)$, for each $x, y \in X(i=1,2, \cdots, m)$. Then we get also similar conclusion case: $p_{i} \in T_{i} p_{i}(i=1,2, \cdots, m)$.

\section{Solution of Integral Equation by VIM}

Recently, the variation iteration method (VIM) has been favorably applied to some various kinds of nonlinear problems, for example, fractional differential equations, nonlinear differential equations, nonlinear thermo elasticity elasticity, nonlinear wave equations.

In this section, we apply the variation iteration method (simple writing VIM) to Integral-differential equations below (see [6,7]). To illustrate the basic idea of the method, we consider:

$$
L[u(t)]+N[u(t)]=g(t) .
$$

The basic character of the method is to construct functional for the system, which reads:

$$
\begin{aligned}
& u_{n+1}(x)=u_{n}(x) \\
& +\int_{0}^{x} \lambda(s) L u\left[L \bar{u}_{n}+N u_{n}-g(s)\right] \mathrm{d} s,
\end{aligned}
$$

which can be identified optimally via variation theory, $u_{n}$ is the nth approximate solution, and $\bar{u}_{n}$ denotes a restricted variation, i.e. $\delta \bar{u}_{n}=0$. There is a iterative formula:

$$
u_{n+1}(x)=f(x)+\lambda \int_{a}^{b} k(x, t) u_{n}(t) \mathrm{d} t
$$

of this equation

$$
u(x)=f(x)+\lambda \int_{a}^{b} k(x, t) u(t) \mathrm{d} t .
$$

Theorem 6.1 (see theorem 3.1 [6]) Consider the iteration scheme $u_{0}(x)=f(x)$, and

$$
u_{n+1}(x)=f(x)+\lambda \int_{a}^{b} k(x, t) u_{n}(t) \mathrm{d} t .
$$

Now, for $n=0,1,2, \cdots$, to construct a sequence of

Successive iterations that for the $\left\{u_{n}(t)\right\}$ for solution of integral Equation $(*)$.

In addition, we assume that

$$
\int_{a}^{b} \int_{a}^{b} k^{2}(x, t) \mathrm{d} x \mathrm{~d} t=B^{2}<\infty,
$$

and $f(x) \in L_{[a, b]}^{2}$, then if $|\lambda|<1 / B$, the above iteration converges in the norm of $L_{[a, b]}^{2}$ to the solution of integral equation $(*)$.

Corollary 6.2 If $k(x, t)=k_{1}(x, t)+k_{2}(x, t)$, and 


$$
\int_{a}^{b} \int_{a}^{b} k^{2}(x, t) \mathrm{d} x \mathrm{~d} t=B^{2}<\infty,
$$

then assume $f(x) \in L_{[a, b]}^{2}$, if $|\lambda|<1 / B$, the above iteration converges in the norm of $L_{[a, b]}^{2}$ to the solution of integral equation $(*)$.

Corollary 6.3 If $k(x, t)=\sum_{i=1}^{m} a_{i}(x) b_{i}(t)$, and

$$
\int_{a}^{b} \int_{a}^{b} k^{2}(x, t) \mathrm{d} x \mathrm{~d} t=B^{2}<\infty,
$$

then assume $f(x) \in L_{[a, b]}^{2}$, if $|\lambda|<1 / B$, the above iteration converges in the norm of $L_{[a, b]}^{2}$ to the solution of integral Equation $(*)$.

Example 6.4 Consider that integral equation

$$
u(x)=x^{\alpha}+x^{\beta}+x+\lambda \int_{0}^{1}(x t+t) u(t) \mathrm{d} t
$$

where

$$
\begin{aligned}
\int_{a}^{b} \int_{a}^{b} k^{2}(x, t) \mathrm{d} x \mathrm{~d} t & =\int_{0}^{1} \int_{0}^{1}(x t+t)^{2} \mathrm{~d} x \mathrm{~d} t \\
& =\frac{1}{9}+\frac{1}{3}+\frac{1}{3}=\frac{7}{9}=B^{2}<\infty,
\end{aligned}
$$

then if $|\lambda|<3 / \sqrt{7}$ (See Figure 1(a)) then the iterative $(*)$ that convergent the solution of Equation (6.2) by corollary 6.2 of theorem 6.1. Therefore, we check that

$$
u_{0}(x)=x^{\alpha}+x^{\beta}+x,(0<\alpha<1,0<\beta<1) .
$$

From (6.1), we have that

$$
u_{n+1}(x)=x^{\alpha}+x^{\beta}+x+\lambda \int_{0}^{1}(x t+t) u_{n}(t) \mathrm{d} t
$$

Let

$$
\begin{aligned}
u_{0}(x) & =x^{\alpha}+x^{\beta}+x, \gamma \\
& =(\alpha+2)^{-1}+(\beta+2)^{-1}+(3)^{-1},(0<|\lambda| \gamma<1), \\
u_{1}(x) & =x^{\alpha}+x^{\beta}+x+\lambda \int_{0}^{1}(x t) u_{0}(t) \mathrm{d} t \\
& =x^{\alpha}+x^{\beta}+\lambda \int_{0}^{1} x\left(t^{\alpha+1}+t^{\beta+1}+t^{2}\right) \mathrm{d} t \\
& =x^{\alpha}+x^{\beta}+x+\lambda \gamma x \\
& =x^{\alpha}+x^{\beta}+x(1+\lambda \gamma),
\end{aligned}
$$

and

$$
\begin{aligned}
u_{2}(x) & =x^{\alpha}+x^{\beta}+x+\lambda \int_{0}^{1}(x t) u_{1}(t) \mathrm{d} t \\
& =x^{\alpha}+x^{\beta}+x \\
& +\lambda x\left((\alpha+2)^{-1}+(\beta+2)^{-1}+(3)^{-1}\right)(1+\lambda \gamma), \\
& =x^{\alpha}+x^{\beta}+x+\lambda \gamma x(1+\lambda \gamma) \\
& =x^{\alpha}+x^{\beta}+x\left(1+\lambda \gamma+(\lambda \gamma)^{2}\right) \\
u_{n}(x) & =x^{\alpha}+x^{\beta}+x\left(1+\lambda \gamma+(\lambda \gamma)^{2}+\cdots+(\lambda \gamma)^{n}\right)
\end{aligned}
$$

(See Figure 1(b), when $n=4$ ).
The exact solution $u(x)=\lim _{n \rightarrow \infty} u_{n}(x)$. Then we obtain exact solution below

$$
\begin{aligned}
u(x) & =x^{\alpha}+x^{\beta}+x\left(1+\lambda \gamma+\lambda^{2} \gamma^{2}+\cdots+\lambda^{n} \gamma^{n}+\cdots\right) \\
& =x^{\alpha}+x^{\beta}+\lambda x(1-\lambda \gamma)^{-1} \text { (See Figure 1(a)). }
\end{aligned}
$$

Remark The exact solution $u(x)$ and approximate solution $u_{4}(x)$ of Example 6.4 (See Figure 1(c)).

Example 6.5 We consider that integral equation

$$
\begin{aligned}
& u(x)=x^{\alpha}+x^{4}+\lambda \int_{0}^{1}\left(x t^{2}+t^{2}\right) u(t) \mathrm{d} t, \\
& u_{0}(x)=x^{\alpha}+x^{4}(0<\alpha<1) .
\end{aligned}
$$

From (6.1), we have that

$$
u_{n+1}(x)=x^{\alpha}+x^{4}+\lambda \int_{0}^{1}\left(x t^{2}+t^{2}\right) u_{n}(t) \mathrm{d} t .
$$

We take

$$
\begin{aligned}
& u_{0}(x)=x^{\alpha}+x^{4}(0<\alpha<1), l=(\alpha+3)^{-1}+(1 / 7) . \\
& u_{n}(x)=x^{\alpha}+x^{4} \\
& +\lambda(x+1)\left(l+\lambda l+\lambda^{2} l^{2}+\lambda^{3} l^{3}+\cdots+\lambda^{n} l^{n}\right) .
\end{aligned}
$$

The exact solution $u(x)=\lim _{n \rightarrow \infty} u_{n}(x)$, then we obtain exact solution below

$$
\begin{aligned}
u(x) & =x^{\alpha}+x^{4} \\
& +\lambda(x+1)\left(l+\lambda l^{2}+\lambda^{2} l^{3}+\cdots+\lambda^{n-1} l^{n}+\cdots\right) \\
& =x^{\alpha}+x^{4}+\lambda I\left((x+1)+\lambda l+(\lambda l)^{2}+\cdots\right) \\
& =x^{\alpha}+x^{4}+\lambda l\left((x+1)+(1-\lambda l)^{-1}\right) .
\end{aligned}
$$

By corollary 6.2 of Theorem 6.1, where that

$$
\int_{a}^{b} \int_{a}^{b} k^{2}(x, t) \mathrm{d} x \mathrm{~d} t=B^{2}<\infty \text {, and }|\lambda|<B^{-1} .
$$

Then the iterative sequence is convergent to the exact solution of the Equation (6.3).

In fact,

$$
\begin{aligned}
\int_{a}^{b} \int_{a}^{b} k^{2}(x, t) \mathrm{d} x \mathrm{~d} t & =\int_{0}^{1} \int_{0}^{1}\left(x t^{2}+t^{2}\right)^{2} \mathrm{~d} x \mathrm{~d} t \\
& =\frac{1}{15}+\frac{1}{5}+\frac{1}{5}=\frac{7}{15}=B^{2}<\infty,
\end{aligned}
$$

then if $|\lambda|<\sqrt{15 / 7}$, the iterative sequence is convergent the solution of Equation (6.3).

Example 6.6 Consider that integral equation ( $0<\alpha<1, \beta>0, \quad k$-positive integer),

$$
u(x)=x^{k \alpha}+\beta x^{2 k}+\lambda \int_{0}^{1}(x t) u(t) \mathrm{d} t,
$$

where $u(0)=0$, and we have that

$$
u_{n}(x)=x^{k \alpha}+\beta x^{2 k}+\lambda \int_{0}^{1}(x t) u_{n-1}(t) \mathrm{d} t
$$

We can take that

$$
u_{0}(x)=x^{k \alpha}+\beta x^{2 k}(0<\alpha<1, \beta>0),
$$




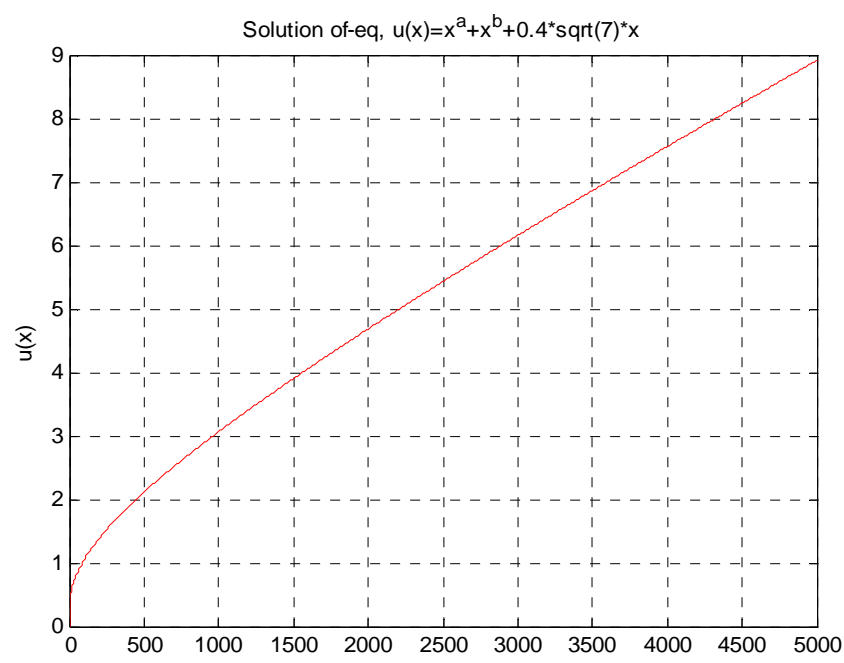

(a)

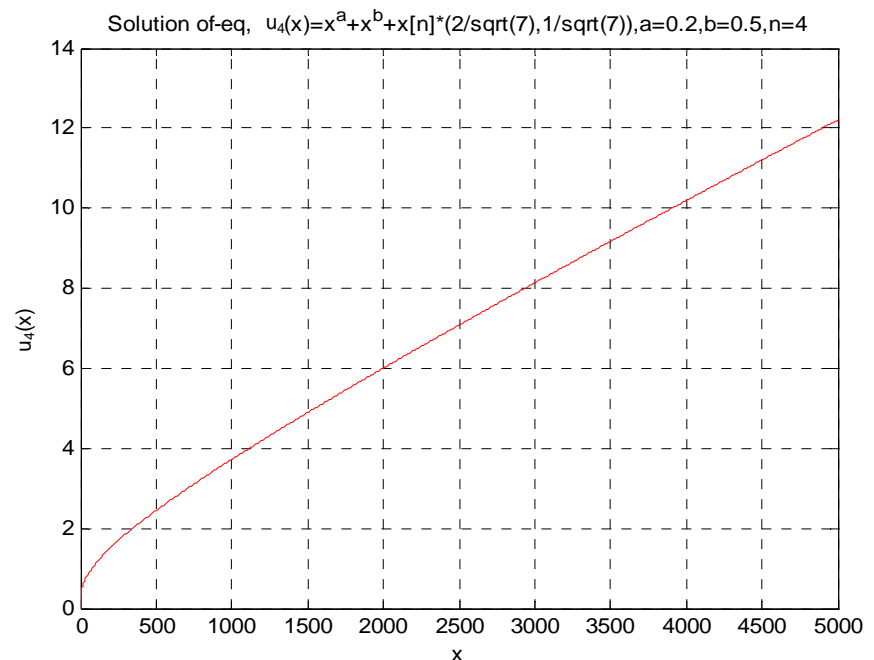

(b)

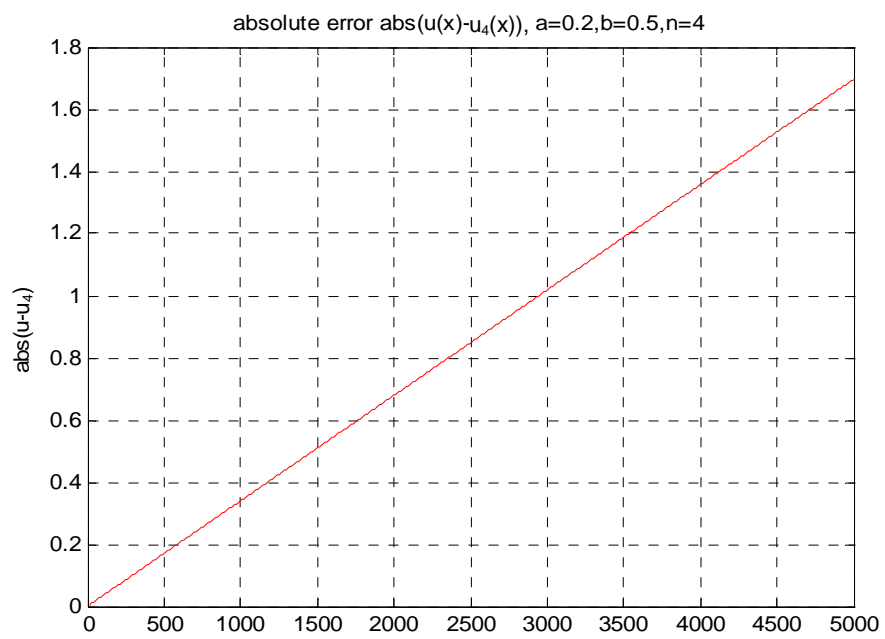

(c)

Figure 1. By Matlab in numerable test. (a) The figure expresses exact solution $u(x)$ for Example 6.4; (b) The figure expresses approximate solution $u_{4}(x)$ for the solution $u(x)$ of Example 6.4; (c) The figure expresses absolute error $\left|u(x)-u_{4}(x)\right|$ for $E x$ ample 6.4. 
( $k$-positive integer), and

$$
\begin{aligned}
u_{1}(x) & =x^{k \alpha}+\beta x^{2 k}+\lambda \int_{0}^{1}(x t) u_{0}(t) \mathrm{d} t=x^{k \alpha}+\beta x^{2 k}+\lambda x \int_{0}^{1}\left(t^{k \alpha+1}+\beta t^{2 k+1}\right) \mathrm{d} t \\
& =x^{k \alpha}+\beta x^{2 k}+\lambda x\left((k \alpha+2)^{-1}+\beta(2 k+2)^{-1}\right) \\
u_{2}(x) & =x^{k \alpha}+\beta x^{2 k}+\lambda \int_{0}^{1}(x t) u_{1}(t) \mathrm{d} t \\
& =x^{k \alpha}+\beta x^{2 k}+\lambda x \int_{0}^{1}\left(t^{k \alpha+1}+\beta t^{2 k+1}+\lambda t^{2}\left((k \alpha+2)^{-1}+\beta(2 k+2)^{-1}\right)\right) \mathrm{d} t \\
& =x^{k \alpha}+\beta x^{2 k}+\left((k \alpha+2)^{-1}+\beta(2 k+2)^{-1}\right)\left(\lambda+(1 / 3) \lambda^{2}\right) x \\
u_{3}(x) & =x^{k \alpha}+\beta x^{2 k}+\lambda \int_{0}^{1}(x t) u_{2}(t) \mathrm{d} t \\
& =x^{k \alpha}+\beta x^{2 k}+\lambda x \int_{0}^{1}\left(t^{k \alpha+1}+\beta t^{2 k+1}+\left((k \alpha+2)^{-1}+\beta(2 k+2)\right)\left(\lambda+(1 / 3) \lambda^{2}\right) t^{2}\right) \mathrm{d} t \\
& =x^{k \alpha}+\beta x^{2 k}+\left((k \alpha+2)^{-1}+\beta(2 k+2)^{-1}\right)\left(\lambda+(1 / 3) \lambda^{2}+(1 / 3)^{2} \lambda^{3}\right) x .
\end{aligned}
$$

Inductively, we have

$$
\begin{aligned}
u_{n}(x) & =x^{k \alpha}+\beta x^{2 k}+\lambda \int_{0}^{1}(x t) u_{n-1}(t) \mathrm{d} t \\
& =x^{k \alpha}+\beta x^{2 k}+\left((k \alpha+2)^{-1}+\beta(2 k+2)^{-1}\right)\left(\lambda+\frac{1}{3} \lambda^{2}+(1 / 3)^{2} \lambda^{3}+\cdots+(1 / 3)^{n-1} \lambda^{n}\right) x
\end{aligned}
$$

Then by Theorem 6.1 and simple computation, we obtain that

$$
\int_{a}^{b} \int_{a}^{b} k^{2}(x, t) \mathrm{d} x \mathrm{~d} t=\int_{0}^{1} \int_{0}^{1}(x t)^{2} \mathrm{~d} x \mathrm{~d} t=\frac{1}{9}=B^{2}<\infty,
$$

then if $|\lambda|<3$, the iterative is convergent the solution of integral equation (6.4) (Similar as examples case in [6, 7]).

\section{Some Effective Modification and Numerical Test for [8]}

In this section, we apply the effective modification method of He's VIM to solve some integral-differential equations. In [6-8] by the variation iteration method (VIM ) simulate the system of this form

$$
L u+R u+N u=g(x) .
$$

To illustrate its basic idea of the method, we consider the following general nonlinear system

$$
L u+R u+N u=g(x), L u
$$

the highest derivative and is assumed easily invertible, $R$ is a linear differential operator of order less than, $\mathrm{Nu}$ represents the nonlinear terms, and $g$ is the source term. Applying the inverse operator $L_{x}^{-1}$ to both sides of above equation, we obtain

$$
u=f-L_{x}^{-1}[R u]-L_{x}^{-1}[N u] .
$$

The variational iteration method (VIM) proposed by
Ji-Huan He (see [6-8] recently has been intensively studied by scientists and engineers. the references cited therein) is one of the methods which have received much concern. It is based on the Lagrange multiplier and it merits of simplicity and easy execution. Unlike the traditional numerical methods. Along the direction and technique in $[4,8]$ we may get more examples below.

Example 7.1 (similar as example in [8]) Consider the following nonlinear Fredholm integral equation

$u^{\prime}(x)-u(x)=x \cos 9 x-x \sin 9 x-\sin 9 x, u(0)=0$.

Applying the inverse operator $L_{x}^{-1}$ to both side of Equation (7.1), yields:

$$
u(x)=x \sin 9 x+\frac{x}{9} \cos 9 x-\frac{1}{81} \sin 9 x+L_{x}^{-1}[u(x)] .
$$

from $L_{x}^{-1}[x \sin x]=-\int_{0}^{x} x d \cos x=\sin x-x \cos x$.

So,

$$
\begin{aligned}
& L_{x}^{-1}[x \sin 9 x]=-\frac{1}{9} \int_{0}^{x} x \mathrm{~d} \cos 9 x=\frac{1}{36} \sin 9 x-\frac{x}{9} \cos 9 x . \\
& \begin{aligned}
u_{1}(x) & =x \sin 9 x+\frac{x}{9} \cos 9 x-\frac{x}{81} \sin 9 x+L_{x}^{-1}\left[f_{0}(x)\right] \\
& =x \sin 9 x .
\end{aligned}
\end{aligned}
$$

Inductively,

$u_{n+1}(x)=x \sin 9 x+\frac{x}{9} \cos x-\frac{1}{81} \sin 9 x+L_{x}^{-1}\left[u_{n}(x)\right]$,

$u_{n+1}(x)=x \sin 9 x, n \geq 1$. 
Then $u(x)=x \sin 9 x$ is exact solution of (7.1). The numerical results are shown in Figure 2.

Example 7.2 Consider the following Volterra-Fredholm integral-differential equation

$u^{\prime}(x)=\mathrm{e}^{x}+\int_{0}^{x}(x+t) u(t) \mathrm{d} t+\int_{0}^{1}(x-t) u^{3}(t) \mathrm{d} t$,

Similar as example1 in this way, we easy get this solution.

According to the method, we divide $f$ into two parts defined $f_{0}(x)=\mathrm{e}^{x}$, and

$$
f_{1}(x)=-p_{3}(x), f(x)=f_{0}(x)+f_{1}(x) .
$$

By calculating this

$$
\begin{aligned}
p_{3}(x)= & \left(\frac{\mathrm{e}^{x}-1}{2}+\frac{\mathrm{e}^{3}-1}{6}\right) x^{2}+\frac{1-2 \mathrm{e}^{3}}{9} x \\
& +\mathrm{e}^{x}(x-1)+1 .
\end{aligned}
$$

So, we have

$$
\begin{aligned}
u(x) & =\mathrm{e}^{x}+L_{x}^{-1}\left[\int_{0}^{x}(x+t) u(t) \mathrm{d} t\right] \\
& +L_{x}^{-1}\left[\int_{0}^{x}(x-t) u^{3}(t) \mathrm{d} t\right]
\end{aligned}
$$

In fact, in this way

$$
\begin{aligned}
& L_{x}^{-1}\left[\int_{0}^{1}(x-t)\left(f_{0}(t)\right)^{3} \mathrm{~d} t\right]=L_{x}^{-1}\left[\int_{0}^{1}\left(x \mathrm{e}^{3 t}-t \mathrm{e}^{3 t}\right) \mathrm{d} t\right] \\
& =\int_{0}^{x}\left(\frac{\mathrm{e}^{3}-1}{3} x-\frac{1}{9}\left(2 \mathrm{e}^{3}-1\right)\right) \mathrm{d} x=\frac{\mathrm{e}^{3}-1}{6} x^{2}-\frac{1}{9}\left(2 \mathrm{e}^{3}-1\right) x,
\end{aligned}
$$

and

$$
\begin{aligned}
L_{x}^{-1}\left(\int_{0}^{x}(x+t) f_{0}(t) \mathrm{d} t\right) & =L_{x}^{-1}\left(\int_{0}^{x}\left(x \mathrm{e}^{t}+t \mathrm{e}^{t}\right) \mathrm{d} t\right) \\
& =\frac{\mathrm{e}^{x}-1}{2} x^{2}+x \mathrm{e}^{x}-\mathrm{e}^{x}+1 .
\end{aligned}
$$

Writing

$$
p_{3}(x)=\left(\frac{\mathrm{e}^{x}-1}{2}+\frac{\mathrm{e}^{3}-1}{6}\right) x^{2}+\frac{1-2 \mathrm{e}^{3}}{9} x+\mathrm{e}^{x}(x-1)+1,
$$

therefore, we have

$$
\begin{aligned}
u_{1}(x)= & \mathrm{e}^{x}-p_{3}(x)+L_{x}^{-1}\left[\int_{0}^{x}(x+t) f_{0}(t) \mathrm{d} t\right] \\
& +L_{x}^{-1}\left[\int_{0}^{1}(x-t) f_{0}(t) \mathrm{d} t\right]=\mathrm{e}^{x}, \cdots
\end{aligned}
$$

Inductively,

$$
\begin{aligned}
u_{n+1}(x)= & \mathrm{e}^{x}-p_{3}(x)+L_{x}^{-1}\left[\int_{0}^{x}(x+t) u_{n}(t) \mathrm{d} t\right] \\
& +L_{x}^{-1}\left[\int_{0}^{1}(x-t) u_{n}(t) \mathrm{d} t\right]=\mathrm{e}^{x},
\end{aligned}
$$

And $u_{n}(x)=\mathrm{e}^{x}$. Hence the $u(x)=\mathrm{e}^{x}$ is the exact solution of (7.2).

Example 7.3 Consider the following integral-differential equation

$$
u^{(5)}(x)=\mathrm{e}^{x}-\frac{4}{3} x+\int_{0}^{1} x t u(t) \mathrm{d} t
$$

where $u(0)=1, u^{\prime}(0)=2, u^{\prime \prime}(0)=1, u^{\prime \prime \prime}(0)=1$ In similar example1, we easy have it .

According to the method, we divide $f$ into two parts defined by

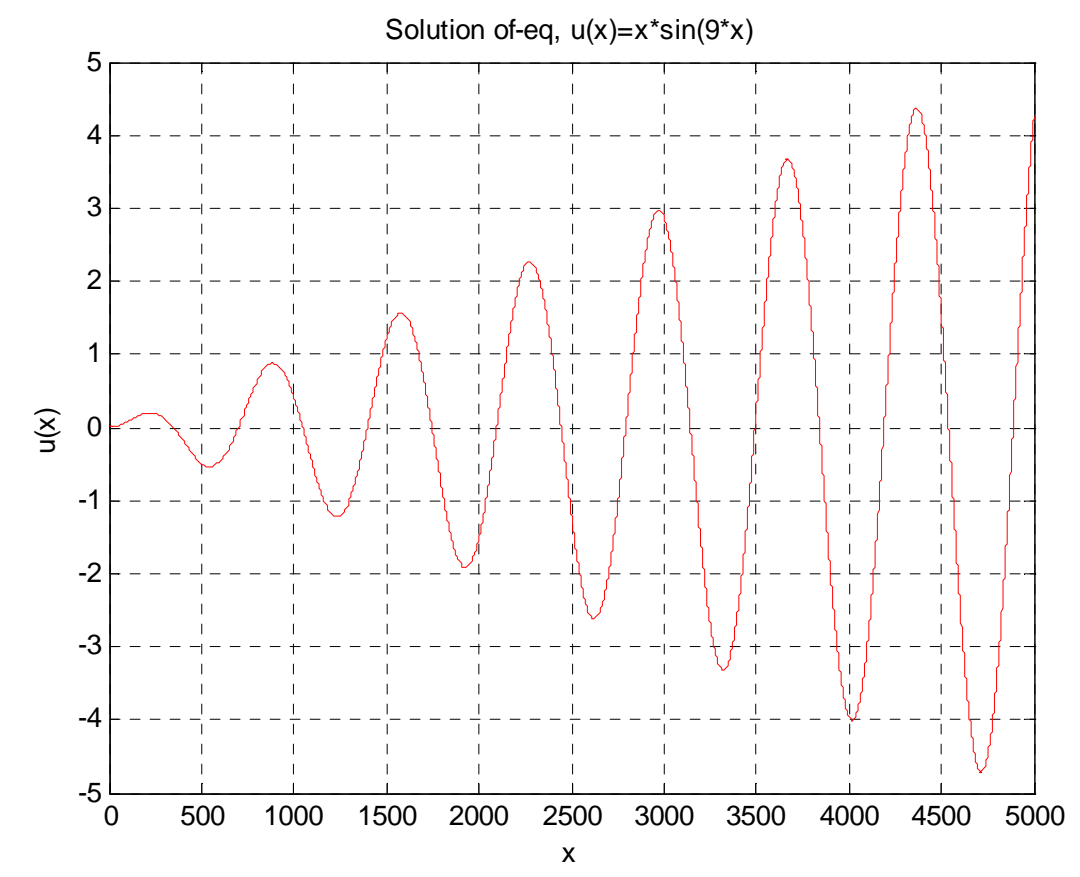

Figure 2. Figures of exact solution $u(x)$ for Example 7.1. 


$$
\begin{aligned}
& f_{0}(x)=x+\mathrm{e}^{x}, u_{0}(x)=f_{0}(x)=x+\mathrm{e}^{x}, \\
& f_{1}(x)=-(4 /(3 * 6 !)) x^{6}=-(2 / 540) x^{6} .
\end{aligned}
$$

Taking $f(x)=x+\mathrm{e}^{x}-(1 / 540) x^{6}$, then we have

$$
\begin{aligned}
u_{1}(x) & =x+\mathrm{e}^{x}-(1 / 540) x^{6}+L_{x}^{-1}\left[\int_{0}^{1} x t f_{0}(t) \mathrm{d} t\right] \\
& =x+\mathrm{e}^{x}
\end{aligned}
$$

where $L_{x}^{-1}(\cdot)=\int_{0}^{x} \int_{0}^{x} \int_{0}^{x} \int_{0}^{x} \int_{0}^{x}(\cdot) \mathrm{d} t \mathrm{~d} t \mathrm{~d} t \mathrm{~d} t \mathrm{~d} t$.

And the processes,

$$
u_{n+1}(x)=x+\mathrm{e}^{x}-(1 / 540) x^{6}+L_{x}^{-1}\left[\int_{0}^{1} x t u_{n}(t) \mathrm{d} t\right], n \geq 1
$$

Thus, $u_{n+1}(x)=x+\mathrm{e}^{x}$, then $u(x)=x+\mathrm{e}^{x}$ is the exact solution of (7.3) by only one iteration leads to a solution. The numerical results are shown in Figure 3.

Example 7.4 (See example 2 in [8]) Consider the following partial differential equation

$$
u_{t}+u u_{x}=a x^{k} t^{k}+b \mathrm{e}^{x} .
$$

Let that integer $k \geq 1,(a, b>0)$, The modified methods:

Applying the inverse operator to both sides of (7.4) yields

$$
u(x, t)=a x^{k} t^{k}+\frac{b}{2 k+1} x^{k} t^{2 k+1}-\frac{1}{2} L_{x}^{-1}\left[u^{2}(x, t)\right]_{x}
$$

where $L_{x}^{-1}(\cdot)=\int_{0}^{x}(\cdot) \mathrm{d} t$.

Here, we divide $f$ into two parts defined by

$$
f_{0}=a x^{k} t^{k}, f_{1}=\frac{1}{2 k+1} x t^{2 k+1}, f(x)=f_{0}(x)+f_{1}(x) .
$$

Using the relation $u_{9}=f_{9}=a x^{k} t^{k}$, we obtain

$$
\begin{aligned}
u_{0}(x, t) & =a x^{k} t^{k}, \\
u_{1}(x, t) & =a x^{k} t^{k}+\frac{b}{2 k+1} x^{k} t^{2 k+1}-\frac{1}{2} L_{x}^{-1}\left[f_{0}^{2}(x, t)\right]_{x} \\
& =a x^{k} t^{k},
\end{aligned}
$$

and so on

$$
\begin{aligned}
u_{n+1}(x, t) & =a x^{k} t^{k}+\frac{b}{2 k+1} x^{k} t^{2 k+1}-\frac{1}{2} L_{x}^{-1}\left[u_{n}^{2}(x, t)\right]_{x} \\
& =a x^{k} t^{k}, n \geq 1 .
\end{aligned}
$$

Hence, $u(x, t)=a x^{k} t^{k}$ is the exact solution of (7.4) and by only one iteration leads to that exact solution. Taking $k=1, a=b=1$ that is example 2 in [8].

The numerical results are shown in Figure 4.

Remark 7.5 Some solving integral-differential equations by VIM may see [9], and that some random Altman type inequality for fixed point results see [10].

The fixed point results of Multi-value mapping are also discussed in [11].

Remark 7.6 By [12], the authors consider the mixed problem for non-linear Burgers equation:

$$
\left\{\begin{array}{l}
\frac{\partial u}{\partial t}+u \frac{\partial u}{\partial x}=\varepsilon \frac{\partial^{2} u}{\partial x^{2}},(x, t) \in(0,1) \times(0,+\infty), \\
u(x, 0)=\phi(x), x \in(0,1) \\
u(0, t)=u(1, t)=0, t \in(0,+\infty) .
\end{array}\right.
$$

The authors point out the problem describes physic phenomenon of motive quality and conservation of law in dynamic problem, it is important model in flow mechanics. Where $u$ express the velocity of flow body,

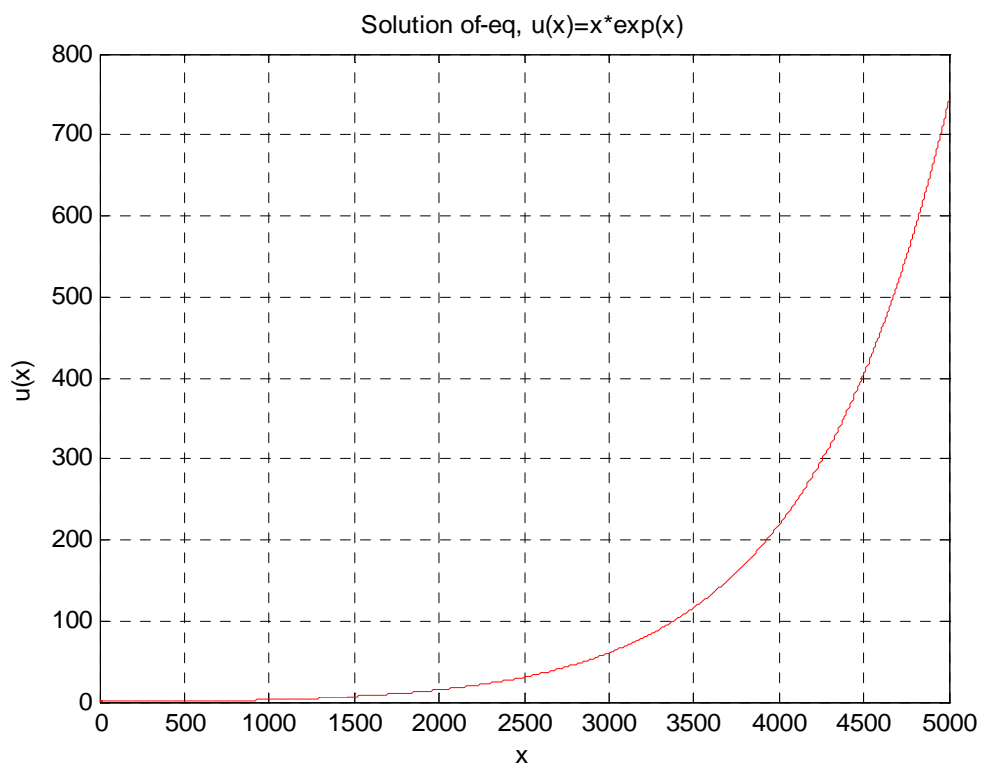

Figure 3. Figures of exact solution $u(x)$ for Example 7.3. 

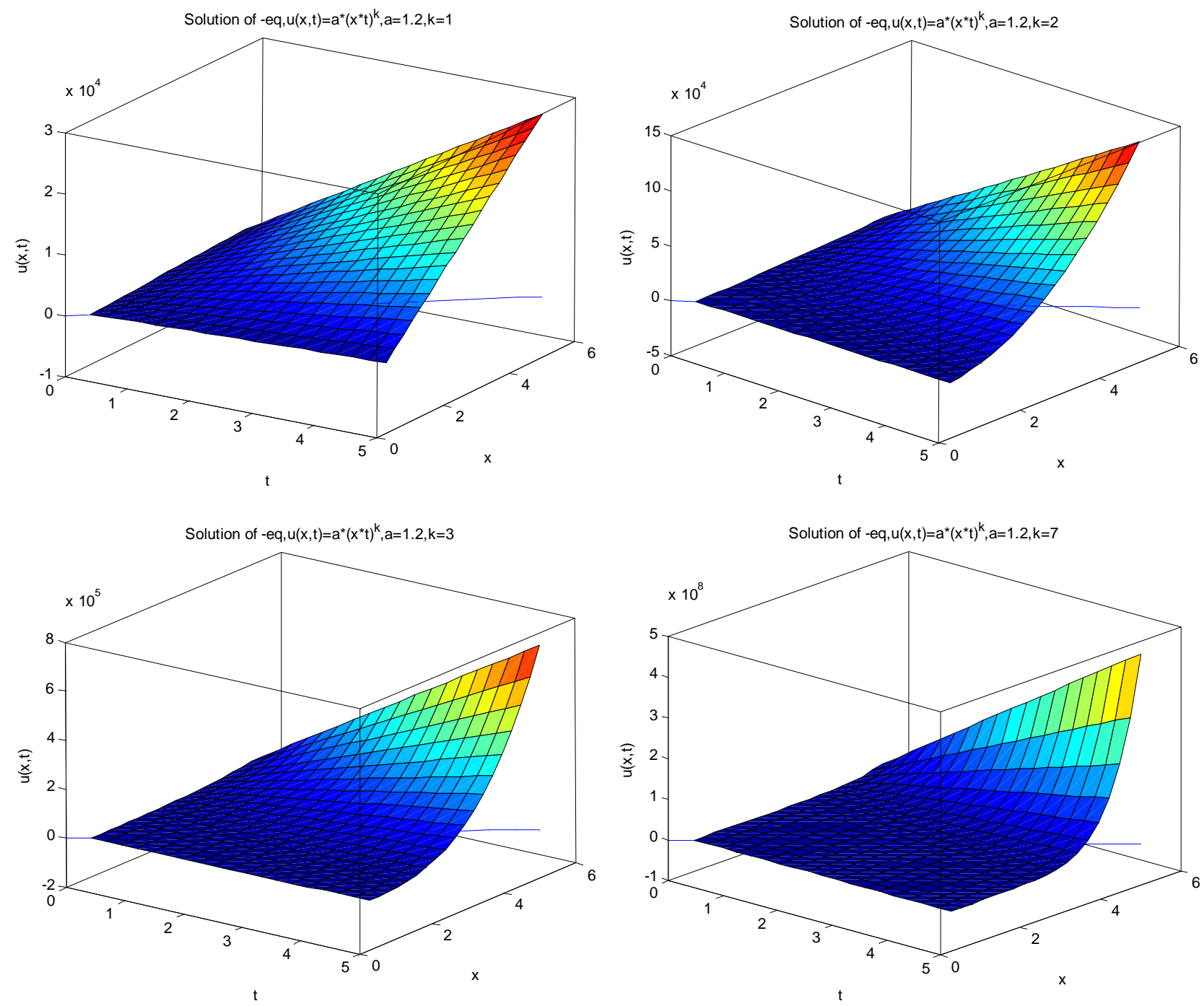

Figure 4. Figures of exact solution $u(x)$ for example 7.4. (where parameters taking as $a=1.2, k=1,2,3,5$ ).

and $\varepsilon$ express the constant of motive flow body,

$\phi(x)$-initial function.

Burger's equation has attracted much attention. The approximation solution for this Burger's equation is also interesting tasks.

\section{Concluding Remarks}

In this Letter, we give out new fixed point theorems in cone metric space and apply the variation iteration method to integral-differential equation, and extend some results in $[3,6-8]$. The obtained solution shows the method is also a very convenient and effective for some various non-linear integral and differential equations, only one iteration leads to exact solutions.

Recently, the impulsive differential delay equation and stochastic schrodinger equation is also a very interesting topic, and may look [11] etc.

\section{Acknowledgements}

This work is supported by the Natural Science Foundation (No.07ZC053) of Sichuan Education Bureau and the key program of Science and Technology Foundation (No.07zx2110) of Southwest University of Science and Technology.

The authors would like to thank the reviewers for the useful comments and some more better results.

\section{REFERENCES}

[1] D. J. Guo and V. Lakshmikantham, "Nonlinear Problems in Abstract Cones,” Academic Press, Inc., Boston, New York, 1988.

[2] L. G. Hung and X. Zhang, "Cone Metric Spaces and Fixed Point Theorems of Contractive Mappings," Journal of Mathematical Analysis and Applications, Vol. 332, No. 2, 2007, pp. 1468-1476. doi:10.1016/j.jmaa.2005.03.087 
[3] X. Zhang, "Common Fixed Point Theorem of Lipschitz Type Mappings on Cone Metric Space," Acta Mathematica Sinica, Chinese Series, Vol. 53, No. 6, 2010, pp. 1139-1148.

[4] H. Avdi, H. K. Nashine, B. Samet and H. Yazidi, "Coincidence and Common Fixed Point Results in Partial Ordered Cone Metric Spaces and Applications to Integral Equations,” Nonlinear Analysis, Vol. 74, No. 17, 2011, pp. 6814-6825. doi:10.1016/j.na.2011.07.006

[5] S. H. Cho and M. S. Kim, "Fixed Point Theorems for General Contractive Multi-Valued Mappings,” Applied Mathematics Information, Vol. 27, No. 1-2, 2009, pp. 343-350.

[6] N. Chen and J. Q. Chen, "New Fixed Point Theorems for 1-Set-Contractive Operators in Banach Spaces," Journal of Fixed Point Theory and Applications, Vol. 6, No. 3, 2011, pp. 147-162.

[7] N. Chen and J. Q. Chen, "Operator Equation and Application of Variational Iterative Method," Applied Mathematics, Vol. 3, No. 8, 2012, pp. 857-863.

doi:10.4236/am.2012.38127
[8] A. Ghorbani and J. Sabaeri-Nadjafi, “An Effective Modification of He's Variational Iteration Method,” Nonlinear Analysis: Real World Applications, Vol. 10, No. 5, 2009, pp. 2828-2833. doi:10.1016/j.nonrwa.2008.08.008

[9] S. Q. Wang and J. H. He, "Variational Iterative Method for Solving Integral-Differential Equations," Physics Letters A, Vol. 367, No. 3, 2007, pp. 188-191. doi:10.1016/j.physleta.2007.02.049

[10] N. Chen, B. D. Tian and J. Q. Chen, "Some Random Fixed Point Theorems and Random Altman Type Inequality," International Journal of Information and Systems Sciences, Vol. 7, No. 1, 2011, pp. 83-91.

[11] S. Jain and V. H. Badshah, "Fixed Point Theorem of Multi-Valued Mappings in Cone Metric Spaces,” International Journal of Mathematical Archive, Vol. 2, No. 12, 2011, pp. 2753-2756.

[12] J. Biazar and H. Aminikahah, "Exact and Numerical Solutions for Non-Linear Burgers's Equation by VIM," Mathematical and Computer Modeling, Vol. 49, No. 7-8, 2009, pp. 1394-1400. doi:10.1016/j.mcm.2008.12.006 